\title{
Efecto del galio sobre la susceptibilidad a la corrosión de una aleación $\mathrm{Ni}-\mathrm{Cr}$ de uso dental ${ }^{(\bullet)}$
}

\author{
O. Riesgo ${ }^{(*)}$, S.M. Vázquez ${ }^{(* *)}$ y G.S. Duffó ${ }^{(* * *)}$ \\ Resumen Las aleaciones Ni-Cr se emplean ampliamente en odontología debido a su buena resistencia a la \\ corrosión en medios orales, a su adecuada adherencia a la cerámica y a su aceptable biocompatibili- \\ dad (aunque este último tópico es un tema seriamente cuestionado en los últimos años). Sin embargo, \\ han aparecido recientemente en el mercado una serie de aleaciones $\mathrm{Ni}-\mathrm{Cr}$ con un contenido de galio \\ del orden del $7 \%$, debido a que esta adición mejoraría las propiedades de la aleación frente a la coc- \\ ción con cerámica. No obstante, no se han llevado a cabo trabajos de laboratorio que demuestren que \\ este material aleado con galio siga presentando buenas propiedades frente a la corrosión como la de \\ los materiales sin adiciones de galio. El objetivo del presente trabajo fue comparar la susceptibilidad \\ a la corrosión de una aleación Ni-Cr con la de una aleación $\mathrm{Ni}-\mathrm{Cr}-\mathrm{Ga}$, en soluciones de iones agresi- \\ vos presentes en la saliva natural. Los resultados mostraron que la presencia de galio no modifica \\ sustancialmente la resistencia a la corrosión de las aleaciones $\mathrm{Ni}-\mathrm{Cr}$.
}

Palabras clave: Aleaciones Ni-Cr. Aleaciones Ni-Cr-Ga. Aleaciones odontológicas. Susceptibilidad a la corrosión.

\section{Effect of gallium on the corrosion susceptibility of an odontological Ni-Cr alloy}

\begin{abstract}
Due to the high price of gold and its alloys, alternative alloys of non noble metals have been developed for use in fixed dental prothesis. These are $\mathrm{Ni}-\mathrm{Cr}$ and $\mathrm{Co}-\mathrm{Cr}$ alloys. Usually fixed prothesis are joined and then covered with a ceramic layer and for this purpose good castability and adherence with ceramics are required. The castability of $\mathrm{Ni}-\mathrm{Cr}$ alloys is good and so is their adherence to the ceramic layer. However, a new Ni-Cr alloy with addition of gallium has been produced. In the present work the corrosion properties of a $\mathrm{Ni}-\mathrm{Cr}-\mathrm{Ga}$ alloy in oral environments are analyzed. The results show that the addition of gallium to a $\mathrm{Ni}-\mathrm{Cr}$ alloy does not produce a modification on the corrosion properties of $\mathrm{Ni}-\mathrm{Cr}$ alloys.
\end{abstract}

Keywords: Ni-Cr alloys. Ni-Cr-Ga alloys. Odontological alloys. Corrosion susceptiblity.

\section{INTRODUCCIÓN}

Debido al alto precio de los metales preciosos y de sus aleaciones, surgió la necesidad de desarrollar aleaciones de metales no nobles para su empleo en

$(\bullet \quad$ Trabajo recibido el día 5 de diciembre de 1995

(*) Fundación para el Estudio de los Materiales Dentales. Alvarado 121, (1704) Ramos Mejía, Buenos Aires (Argentina).

(**) Laboratorio de Sólidos Amorfos. Dpto. de Física. Facultad de Ingeniería. Universidad de Buenos Aires. Paseo Colón 810, (1063), Buenos Aires (Argentina).

(***) Comisión Nacional de Energía Atómica. Gerencia Centro Atómico Constituyentes. Unidad de Actividad Materiales. Av. del Libertador 8250, (1429), Buènos Aires (Argentina). la fabricación de prótesis dentales. Dentro de estas aleaciones, una de las empleadas más frecuentemente debido a su buena resistencia mecánica, alto módulo elástico, baja densidad y alta resistencia a la corrosión, es la aleación Ni-Cr. Otra de las propiedades remarcables de esta aleación es su buena adherencia a la cerámica. No obstante, y desde hace algunos años, en un intento por acentuar aún más esta propiedad, se adiciona a las aleaciones $\mathrm{Ni}-\mathrm{Cr}$ una determinada cantidad de galio que, además de disminuir el punto de fusión de la aleación por las características de su óxido, aumenta las interacciones químicas y mecánicas con la cerámica, incrementando de esta manera la fuerza de adhesión cerámica-aleación (1). 
El empleo del galio como constituyente de aleaciones dentales es reciente, y se ha introducido como alternativa al empleo de amalgamas dentales debido a la toxicidad del mercurio (2). Sin embargo, se ha encontrado que las aleaciones de base galio presentan escasa resistencia a la corrosión en cavidades orales (3). Por este motivo, resulta de particular interés estudiar el efecto que tiene el agregado de dicho metal sobre la susceptibilidad a la corrosión de las aleaciones odontológicas $\mathrm{Ni}-\mathrm{Cr}$.

El objetivo del presente trabajo fue evaluar el comportamiento frente a la corrosión de una aleación $\mathrm{Ni}-\mathrm{Cr}-\mathrm{Ga}$ de uso odontológico, y compararla con el de una aleación Ni-Cr. Esta última aleación ha sido ampliamente estudiada, y los resultados obtenidos fueron objeto de diferentes publicaciones (4-6).

\section{TÉCNICA EXPERIMENTAL}

Se estudiaron dos aleaciones comerciales ( $\mathrm{Ni}-\mathrm{Cr}$ y Ni-Cr-Ga) cuya composición química, determinada por espectrometría de absorción atómica y por gravimetría, se muestra en la tabla I. Estas aleaciones se fundieron mediante una llama de oxígenopropano-butano, y las probetas se obtuvieron por el método de la cera perdida. Las probetas coladas eran placas de aproximadamente $2 \times 1 \times 0,2 \mathrm{~cm}$, con un pequeño agujero en uno de sus extremos a fin de realizar el contacto eléctrico correspondiente.

Las probetas así obtenidas se desbastaron suavemente con alúmina y se montaron adecuadamente para efectuar estudios electroquímicos. Se estableció en cada una un contacto eléctrico a través de un alambre de cobre que pasaba por un tubo de vidrio $\mathrm{y}$, posteriormente, se cubrieron con resina epoxi, dejando un área libre expuesta de aproximadamente $1 \mathrm{~cm}^{2}$. Las muestras así montadas se desengrasaron con acetona y se secaron con aire caliente. Los ensayos electroquímicos (curvas de polarización) se realizaron en una solución de saliva artificial simi-

Tabla I.- Composición química (p/p) de las aleaciones $\mathrm{Ni}-\mathrm{Cr}$ y $\mathrm{Ni}-\mathrm{Cr}-\mathrm{Ga}$ empleadas en el presente trabajo

TABLE I.- Chemical composition (w/o) of $\mathrm{Ni}$ - $\mathrm{Cr}$ and $\mathrm{Ni}-\mathrm{Cr}$-Ga alloys used in the present work

\begin{tabular}{|c|c|c|}
\hline Elemento & Aleación Ni-Cr & Aleación Ni-Cr-Ga \\
\hline $\mathrm{Ni}$ & 72,26 & 66,80 \\
$\mathrm{Cr}$ & 13,52 & 12,50 \\
$\mathrm{Mo}$ & 8,11 & 7,50 \\
$\mathrm{Si}$ & 1,00 & 0,70 \\
$\mathrm{Fe}$ & 5,11 & 4,72 \\
$\mathrm{Ga}$ & - & 7,78 \\
\hline
\end{tabular}

lar a la sugerida por Ross et al. (7) $\left(\mathrm{K}_{2} \mathrm{HPO}_{4} 0,20\right.$ $\mathrm{g} / \mathrm{l} ; \mathrm{Na}_{2} \mathrm{HPO}_{4}$ 0,26 g/l; KCNS 0,33 g/l; $\mathrm{NaHCO}_{3}$ $1,50 \mathrm{~g} / \mathrm{l} ; \mathrm{NaCl} 0,70 \mathrm{~g} / \mathrm{l} ; \mathrm{KCl} 1,20 \mathrm{~g} / \mathrm{l}$ y urea $0,13 \mathrm{~g} / \mathrm{l}$. El valor de $\mathrm{pH}$ se ajustó a 7,6 con adición de ácido láctico) y en una solución $0,1 \mathrm{M}$ de $\mathrm{NaCl}+0,1 \mathrm{M}$ ácido láctico $(\mathrm{pH} \mathrm{2,3).} \mathrm{Estas} \mathrm{soluciones} \mathrm{se} \mathrm{prepara-}$ ron con reactivos de grado analítico y agua de alta pureza (18 $\mathrm{M} \Omega \cdot \mathrm{cm}$ de resistividad). Los potenciales de electrodo que se dan en este trabajo se determinaron empleando un electrodo de calomelanos saturado (ECS), y con referencia a la escala del electrodo normal de hidrógeno (ENH).

Los ensayos consistieron en el trazado de las curvas de polarización potenciocinéticas (por triplicado), comenzando a un potencial $50 \mathrm{mV}$ por debajo del correspondiente potencial de corrosión $\left(E_{\text {corr }}\right)$, a una velocidad de barrido de $0,5 \mathrm{mV} \cdot \mathrm{s}^{-1}$. Antes de introducir las probetas en la solución se desairearon durante 90 min por medio del burbujeo de nitrógeno purificado. Previamente al inicio de las mediciones, se permitía que las muestras alcanzaran un potencial de corrosión cuasi estacionario, por exposición a la solución a temperatura ambiente durante $1 \mathrm{~h}$ a circuito abierto bajo burbujeo de nitrógeno. Las medidas se llevaron a cabo a temperatura ambiente con un potenciostato PAR 176 y un equipo de barrido de potenciales PAR 175, obteniéndose un registro gráfico de las curvas mediante un equipo Houston X-Y Recorder. Para los ensayos se empleó una celda convencional de tres electrodos con una capacidad de $50 \mathrm{~cm}^{3}$, que ya se describió en una publicación previa (4). Tras los ensayos, las probetas se observaron mediante microscopio óptico y con un microscopio electrónico de barrido Philips SEM 500.

\section{RESULTADOS Y DISCUSIÓN}

En la figura 1 se muestran las curvas de polarización obtenidas en solución de saliva artificial. Se puede apreciar que el comportamiento de las dos aleaciones ensayadas ( $\mathrm{Ni}-\mathrm{Cr}$ y $\mathrm{Ni}-\mathrm{Cr}-\mathrm{Ga}$ ) es similar: tras el potencial de corrosión (aproximadamente $0,100 \mathrm{~V}(\mathrm{ENH})$ ), la corriente sube lentamente hasta llegar a una zona pasiva con intensidades menores de $0,1 \mu \mathrm{A} / \mathrm{cm}^{2}$. Dicha pasividad se debe a la presencia de iones fosfato que inducen a la formación de fosfatos de níquel, fenómeno observado en diversas aleaciones de níquel ( 5 y 8 ). La zona pasiva se extiende hasta los $0,320 \mathrm{~V}(\mathrm{ENH})$, donde la corriente sube abruptamente debido a la ruptura de la pasividad.

Después del trazado de las curvas de polarización, la observación microscópica de las probetas reveló la presencia de picaduras profundas que se iniciaban preferentemente en las zonas interdendríticas. 


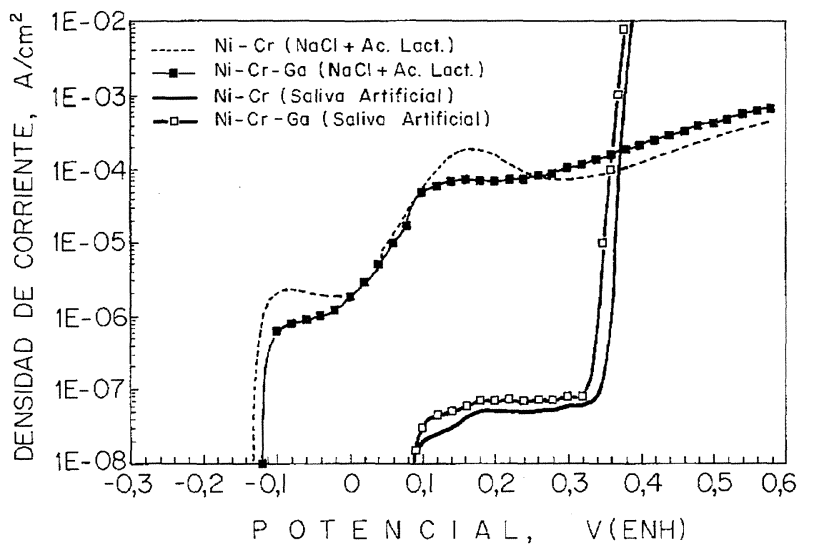

FIG. 1.- Curvas de polarización de las aleaciones $\mathrm{Ni}-\mathrm{Cr}$ y Ni-Cr-Ga, en solución de saliva artificial y en solución $\mathrm{NaCl} 0,1 \mathrm{M}$ + ácido láctico $0,1 \mathrm{M}$.

FIG. 1.- Anodic polarization curves of $\mathrm{Ni}-\mathrm{Cr}$ and $\mathrm{Ni}-\mathrm{Cr}-\mathrm{Ga}$ alloys in artificial saliva and $0.1 \mathrm{M} \mathrm{NaCl}$ + O.1M lactic acid solutions.

Debido a la similitud encontrada entre el comportamiento electroquímico de las aleaciones $\mathrm{Ni}-\mathrm{Cr}$ y Ni-Cr-Ga en solución de saliva artificial, se procedió a ensayar dichas aleaciones en un medio más agresivo que contenía únicamente iones cloruro y ácido láctico. Los resultados obtenidos se muestran en la figura 1. Nuevamente, se puede observar que el potencial de corrosión de ambas aleaciones coincide $(-0,120 \mathrm{~V}(\mathrm{ENH}))$. A partir del mismo la densidad de corriente sube hasta alcanzar una zona pasiva de aproximadamente $100 \mathrm{mV}$ de extensión con corrientes del orden de algunos $\mu \mathrm{A} / \mathrm{cm}^{2}$ (más de un orden de magnitud mayor que la corriente de pasividad encontrada en medios conteniendo fosfatos) que posteriormente se incrementa al aumentar el potencial, presentando un máximo local a aproximadamente $0,150 \mathrm{~V}(\mathrm{ENH})$ para luego volver a subir monótonamente con el aumento del potencial aplicado.

En algunos ensayos, al llegar 0,600 V(ENH) durante el trazado de las curvas de polarización, se dejaron las probetas sometidas a dicho potencial durante un tiempo de aproximadamente $1 \mathrm{~h} 30 \mathrm{~min}$, para luego observarlas al microscopio electrónico de barrido. En dicha observación se dedujo que ambas aleaciones sufren picado bajo formas irregulares (Fig. 2) y que el material es altamente susceptible a la corrosión por rendijas (Fig. 3).

El cálculo de las velocidades de corrosión en solución de saliva artificial se llevó a cabo mediante un método convencional ( 9 y 10 ) a partir de la resistencia de polarización y de la pendiente de Tafel anódica, suponiendo que la reducción de oxígeno es el proceso parcial catódico, si bien el proceso global está bajo control anódico al imponer la cinética las características de la capa pasiva. Los

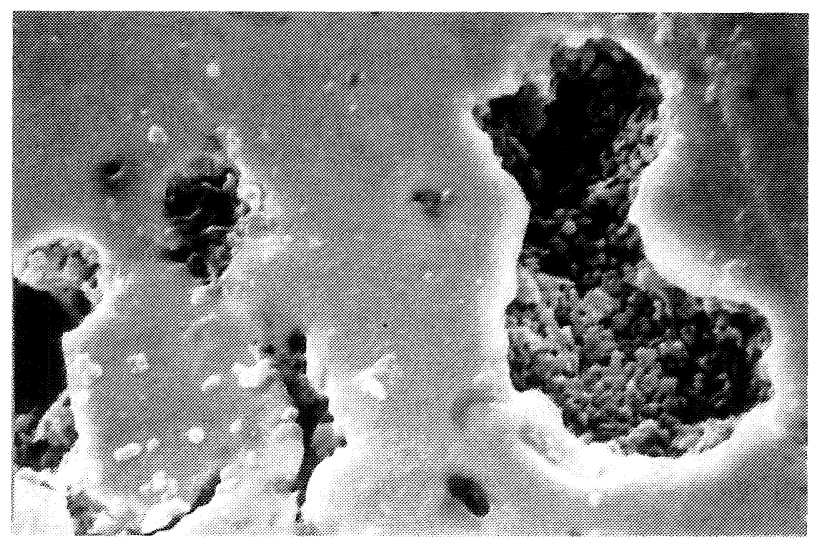

FIG. 2.- Aspecto superficial de una probeta de NiCr-Ga después del trazado de la curva de polarización en solución $\mathrm{NaCl} 0,1 \mathrm{M}+$ ácido láctico $0,1 \mathrm{M}, \mathrm{y}$ de mantenerla durante 1 h 30 min a $0,600 \mathrm{~V}(\mathrm{ENH})$. Se observa profusa distribución de picaduras irregulares. $\times 1.200$.

FIG. 2.-Pits formed on a Ni-Cr-Ga specimen after anodic polarization in $0.1 \mathrm{M} \mathrm{NaCl}+0.1 \mathrm{M}$ lactic acid solution. Potential scanning plus $1.5 \mathrm{~h}$ exposition at $0.6 \mathrm{~V}(\mathrm{NHE}) . \times 1.200$.

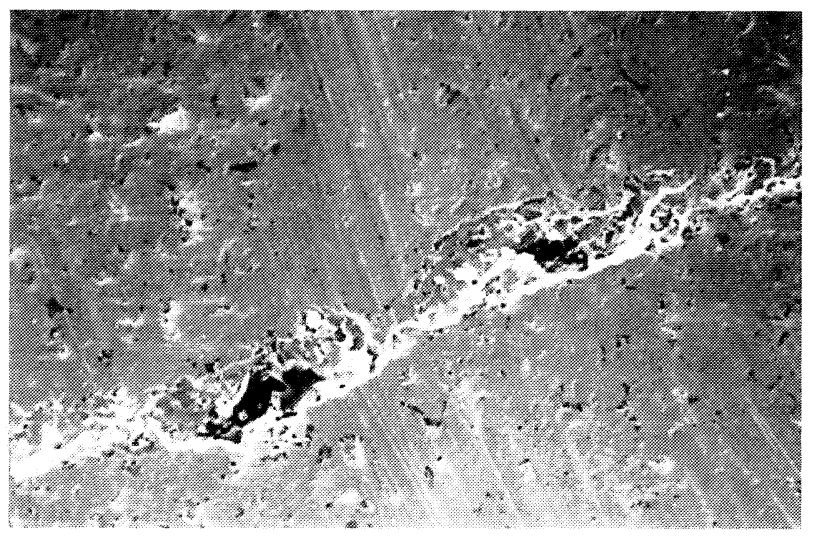

FIG. 3.- Aspecto superficial de una probeta de NiCr-Ga que presenta el fenómeno de corrosión por rendijas tras el trazado de la curva de polarización y de mantenerla a $0,6 \mathrm{~V}(\mathrm{ENH})$ durante $1 \mathrm{~h} 30 \mathrm{~min}$ en solución de $\mathrm{NaCl} 0,1 \mathrm{M}$ + ácido láctico $0,1 \mathrm{M}$. $\times 150$.

FIG. 3.- Crevice corrosion observed on a $\mathrm{Ni}-\mathrm{Cr}$ $\mathrm{Ga}$ specimen after anodic polarization in $0.1 \mathrm{M}$ $\mathrm{NaCl}+0.1 \mathrm{M}$ lactic acid solution. Potential scanning plus $1.5 \mathrm{~h}$ exposition at $0.6 \mathrm{~V}(\mathrm{NHE})$. $\times 150$.

cálculos determinaron que la aleación $\mathrm{Ni}-\mathrm{Cr}-\mathrm{Ga}$ presenta una velocidad de corrosión del orden de $4 \cdot 10^{-3} \mathrm{~mm} / \mathrm{año}$, comparable con la determinada para la aleación $\mathrm{Ni}-\mathrm{Cr}$ (6 y 10). Este valor es lo suficientemente bajo como para aceptar el empleo de las aleaciones Ni-Cr-Ga en la construcción de prótesis dentales. 


\section{CONCLUSIONES}

Como conclusión del presente trabajo, se puede establecer que la presencia del galio no modifica sustancialmente la resistencia a la corrosión de las aleaciones $\mathrm{Ni}-\mathrm{Cr}$ en soluciones que simulen los medios orales, que el fenómeno electroquímico observado consiste en la presencia de una zona pasiva seguida de un potencial de ruptura a partir del cual el material sufre picado y que las velocidades de corrosión involucradas son lo suficientemente bajas como para que dicha aleación pueda ser considerada apta para su uso en medios orales. Sin embargo, al no haberse podido encontrar evidencia experimental favorable con respecto a que la presencia de galio mejore la adherencia a la porcelana (11), en el balance final se encuentra que, aparentemente, la presencia de galio no produce beneficios significativos cuando se adiciona a las aleaciones $\mathrm{Ni}-\mathrm{Cr}$ tradicionales.

\section{REFERENCIAS}

(1) Mueller, H.J. Tarnish and Corrosion of Dental Alloy. ASM Handbook, Vol. 13 (Corrosion). ASM International 9th Ed. 1992: 1.354.
(2) Broome, J.C., Osborne, J.W., Lacefield, W.R. y LeinFELDER, K.F. J. Dent. Res., 74, 1995: 103.

(3) Kaga, M., Sakai, T. y Oguchi, H. J. Dent. Res., 74, 1995: 104.

(4) De Micheli, S.M. DE y Riesgo, O. Rev. D'Odonto-Stomatologie, 7, 1978: 349-353.

(5) De Micheli, S.M. DE y Riesgo, O. Biomater., 3, 1982: 209-212.

(6) Riesgo, O., BiAnchi, G. y Duffo, G.S. Rev. Asoc. Odontol. Argenti., 79, 1992: 6-9.

(7) Ross, T.K., Carter, D.A. y Smith, D.C. Corros. Sci., 7, 1967: 373-380.

(8) Mc Cright, R.D. y Staehle, R.W. Corrosion and stress corrosion cracking of materials for water cooled reactors. Electric Power Research Institute. Project RP311-1. Semi-Anual Progress Report 1975-1976. Ohio State University RSPD (EE.UU.), 4152 A1.

(9) Gonzalez Fernandez, J.A. Teoría y práctica de la lucha contra la corrosión. Ed. CSIC. Madrid, 1984: 629.

(10) Riesgo, O., Bianchi, G.L. y Duffo, G.S. Rev. Metal. CENIM, 28 (4), 1992: 215-218.

(11) Riesgo, O., Palacios, T. y Vigna, G. Les Cah. Prothese, 42, 1983: 95-97. 